\title{
Almost structural completeness; an algebraic approach
}

\author{
Wojciech Dzik ${ }^{1}$ and Michał M. Stronkowski ${ }^{2}$ \\ 1 University of Silesia, Katowice, Poland \\ dzikw@ux2.math.us.edu.pl \\ 2 Warsaw University of Technology, Warsaw, Poland \\ m.stronkowski@mini.pw.edu.pl
}

\begin{abstract}
The notion of structural completeness has received considerable attention for many years. A translation to algebra gives: a quasivariety is structurally complete if it is generated by its free algebras. It appears that many deductive systems (quasivarieties), like $\mathrm{S} 5$ or $\mathrm{MV}_{n}$ fails structural completeness for a rather immaterial reason. Therefore the adjusted notion was introduced: almost structural completeness. We investigate almost structural completeness from an algebraic perspective and obtain a characterization of this notion for quasivarieties.

By a deductive system we mean a pair $\mathcal{S}=(A, R)$, where $A$ is a set of axioms and $R$ is a set of inference rules. Let $\operatorname{Th}(\mathcal{S})$ be the set of theorems derivable in $\mathcal{S}$. A (structural) inference rule is admissible in $\mathcal{S}$ if whenever applied to sentences in $\operatorname{Th}(\mathcal{S})$ it outputs a sentence in $\operatorname{Th}(\mathcal{S})$. A deductive system $\mathcal{S}$ is structurally complete if every inference rule admissible in $\mathcal{S}$ is derivable in $\mathcal{S}$ [4]. Informally, one may rephrase it as follows: Whatever may be added to improve the proof strength of a deductive system is already derivable in it.

For algebraizable deductive systems in the sense of [2] there is a very nice characterization of structural completeness. Every algebraizable deductive system $\mathbf{S}=(A, R)$ has a quasivariety counterpart $\mathcal{Q}$. Logical connectives of $\mathcal{S}$ become basic operations for $\mathcal{Q}, A$ and $R$ translates to an axiomatization of $\mathcal{Q}: A$ to identities, and $R$ to quasi-identities. Then $\mathcal{S}$ is structurally complete if and only if $\mathcal{Q}$ is generated by $\mathbf{F}$ a free algebra in $\mathcal{Q}$ of denumerable rank. Thus structural completeness may be investigated algebraically [1].

Sometimes it happens that a deductive system $\mathcal{S}$ is not structurally complete, but admissible non-derivable inference rules cannot be applied to sentences in $\operatorname{Th}(\mathcal{S})$. Thus adding these inference rules does not improve the proof strength of $\mathcal{S}$ anyway. Such deductive systems are as good as structurally complete deductive systems, and we call them almost structurally complete.

Let us give an example in modal logic, where the advantage of dealing with almost structural completeness instead of structural completeness is particularly apparent. Let $\mathcal{S}=(A, R)$ be a modal deductive system with $R$ consisting of necessitation and modus ponens. Then the algebraic counterpart of $\mathcal{S}$ is a variety $\mathcal{V}$ of modal algebras defined by identities obtained from $A$. Assume that in $\mathcal{V}$ there is an algebra $\mathbf{M}$ with more than 2 elements and satisfying $\diamond x=1$ for all $x \neq 0$ and $\diamond 0=0$. Equivalently, $A$ holds in some nontrivial frame with total relation. Then $\mathcal{S}$ cannot be structurally complete. Indeed, consider the inference rule
\end{abstract}

$$
\frac{\diamond p \wedge \diamond \neg p}{\perp} .
$$

Since in a two-element modal algebra $(\forall x)[\diamond x \wedge \diamond \neg x \not \approx 1]$ holds, the sentence $\diamond \varphi \wedge \diamond \neg \varphi$ is not in $\operatorname{Th}(\mathcal{S})$ for every modal formula $\varphi$, and hence $(\mathrm{r})$ is admissible in $\mathcal{S}$. But it is not derivable in $\mathcal{S}$ for $\mathbf{M}$ does not satisfy the quasi-identity $(\forall x)[\nabla x \wedge \diamond \neg x \approx 1 \rightarrow 0 \approx 1]$. There are many modal deductive systems of this kind which however are almost structurally complete. The best 
known among them is S5 logic, i.e., the logic of equivalence relations, considered as a deductive system.

In this project our aim is to investigate almost structural completeness from the algebraic point of view, in particular, to obtain an algebraic characterization of this notion. Let us formulate a definition of almost structural completeness for quasivarieties precisely. For a quasivariety $\mathcal{Q}$ let $\mathbf{F}$ denote a free algebra in $\mathcal{Q}$ of denumerable rank, and $Q(\mathbf{F})$ the quasivariety generated by $\mathbf{F}$. Recall that $\mathcal{Q}$ is structurally complete iff $\mathcal{Q}=\mathrm{Q}(\mathbf{F})$. For a quasi-identity

$$
q=(\forall \bar{x})\left[s_{1}(\bar{x}) \approx t_{1}(\bar{x}) \wedge \cdots \wedge s_{n}(\bar{x}) \approx t_{n}(\bar{x}) \rightarrow s(\bar{x}) \approx t(\bar{x})\right]
$$

let

$$
q^{*}=(\forall \bar{x})\left[s_{1}(\bar{x}) \not t_{1}(\bar{x}) \vee \cdots \vee s_{n}(\bar{x}) \not \approx t_{n}(\bar{x})\right] .
$$

Definition. We say that a quasivariety $\mathcal{Q}$ is almost structurally complete provided for every quasi-identity $q$ which is valid in $\mathbf{F}$ but not in $\mathcal{Q}$, the sentence $q^{*}$ is valid in $\mathbf{F}$.

We obtained the following characterization.

Theorem 1. The following conditions are equivalent:

1. $\mathcal{Q}$ is almost structurally complete;

2. For every algebra $\mathbf{A} \in \mathcal{Q}, \mathbf{A} \times \mathbf{F} \in \mathrm{Q}(\mathbf{F})$;

3. For every relatively subdirectly irreducible algebra $\mathbf{A} \in \mathcal{Q}, \mathbf{A} \times \mathbf{F} \in \mathrm{Q}(\mathbf{F})$;

4. For every algebra $\mathbf{A} \in \mathcal{Q}$, the existence of a homomorphism $h: \mathbf{A} \rightarrow \mathbf{F}$ yields $\mathbf{A} \in \mathbf{Q}(\mathbf{F})$;

5. For every finitely presented algebra $\mathbf{A} \in \mathcal{Q}$, the existence of a homomorphism $h: \mathbf{A} \rightarrow \mathbf{F}$ yields $\mathbf{A} \in \mathbf{Q}(\mathbf{F})$.

Besides Theorem 1, we obtained a fairly nice characterization of almost structural completeness under some additional assumptions.

Theorem 2. Let $\mathcal{Q}$ be a quasivariety in a finite language with finite model property and equationally definable principal relative congruences. Assume that $\mathbf{F}$ has a finite simple subalgebra C. Then $\mathcal{Q}$ is almost structurally complete if and only if for every finite relatively subdirectly irreducible algebra $\mathbf{S} \in \mathcal{Q}$ we have

$$
\mathbf{S} \leqslant \mathbf{F} \quad \text { or } \quad \mathbf{S} \times \mathbf{C} \leqslant \mathbf{F} .
$$

Let us emphasize that all assumptions in Theorem 2 are very natural from the perspective of logic. Indeed, when $\mathcal{Q}$ is the algebraic counterpart of a deductive system $\mathcal{S}=(A, R)$, then the finite model property with the finiteness of $A$ guarantee the decidability of $\operatorname{Th}(\mathcal{S})$. Moreover, $\mathcal{Q}$ has equationally definable principal relative congruences if and only if $\mathcal{S}$ has the deduction-detachment theorem [3, Theorem 5.5]. Finally, $\mathbf{C}$ may be often chosen as a twoelement Boolen algebra, with possibly additional operations, where the elements correspond to verum and falsum.

During a talk we will provide examples illustrating both theorems.

\section{References}

[1] Clifford Bergman. Structural completeness in algebra and logic. In Algebraic logic (Budapest, 1988), volume 54 of Colloq. Math. Soc. János Bolyai, pages 59-73. North-Holland, Amsterdam, 1991. 
[2] W. J. Blok and Don Pigozzi. Algebraizable logics. Mem. Amer. Math. Soc., 77(396):vi+78, 1989.

[3] Willem J. Blok and Don Pigozzi. Abstract algebraic logic and the deduction theorem, 2001. Manuscript available at http://orion.math.iastate.edu/dpigozzi/.

[4] W. A. Pogorzelski. Structural completeness of the propositional calculus. Bull. Acad. Polon. Sci. Sér. Sci. Math. Astronom. Phys., 19:349-351, 1971. 